Original Article

\title{
ACUTE AND SUB CHRONIC TOXICITY STUDIES OF PURIFIED WITHANIA SOMNIFERA EXTRACT IN RATS
}

\author{
BENNY ANTONY*, MERINA BENNY, BINU T. KURUVILLA, NISHANT KUMAR GUPTA, ANU SEBASTIAN, SHERINA \\ JACOB
}

Arjuna Natural Ltd. (formerly Arjuna Natural Extracts Ltd.), Research and Development Laboratory, Door No.187 D, Behind ISRO, Erumathala P. 0, Keezhmad, Aluva, Kerala-683112, India

Email: research@arjunanatural.com

Received: 03 Sep 2018 Revised and Accepted: 27 Oct 2018

\begin{abstract}
Objective: The objective of the present study was to evaluate the acute and sub-chronic ( $90 \mathrm{~d}$; repeated dose) toxicity of Withania somnifera (ashwagandha) extract in rats.

Methods: The acute toxicity was evaluated as per OECD (Organisation for Economic Co-operation and Development) guidelines 423. Purified ashwagandha extract (PAE) was fed at $2000 \mathrm{mg} / \mathrm{kg}$ body weight (bw) to overnight fasted female rats. The animals were observed daily for clinical signs of abnormality/mortality. After $14 \mathrm{~d}$, animals were sacrificed and gross pathological changes were recorded. Sub-chronic toxicity of PAE was studied by feeding the extract at 100,500 and $1000 \mathrm{mg} / \mathrm{kg}$ bw daily to rats as per OECD guidelines 408 . After $90 \mathrm{~d}$ feeding, heamatological and biochemical parameters of treated rats were compared with control animals. Histopathology of all the major organs was also studied.

Results: In the acute toxicity study, no mortality or clinical signs of toxicity were observed in any of the animals at maximum recommended dose level of $2000 \mathrm{mg} / \mathrm{kg} \mathrm{bw}$; therefore the LD50 is $>2000 \mathrm{mg} / \mathrm{kg}$ bw in rats. The repeated administration of PAE for $90 \mathrm{~d}$ in rats at the maximum dose level of $1000 \mathrm{mg} / \mathrm{kg}$ bw did not induce any observable toxic effects, when compared to its corresponding control animals. The hematology and biochemistry profile of treated rats was similar to control animals and difference was non-significant ( $p>0.05$ ). The histopathology of major organs of all the control and treated animals was normal. In this study the NOAEL (No Observed Adverse Effect Level) was calculated as $1000 \mathrm{mg} / \mathrm{kg}$ bw daily for rats.
\end{abstract}

Conclusion: The present study clearly indicates that PAE does not have any toxic effects in animals at the dose evaluated as evidenced by acute and sub chronic toxicity studies in rats.

Keywords: OECD 423, OECD 408, Toxicity study, Withania somnifera; Shoden

(c) 2018 The Authors. Published by Innovare Academic Sciences Pvt Ltd. This is an open access article under the CC BY license (http://creativecommons.org/licenses/by/4.0/) DOI: http://dx.doi.org/10.22159/ijpps.2018v10i12.29493

\section{INTRODUCTION}

Withania somnifera (ashwagandha) belongs to family Solanaceae is commonly known as "Indian Ginseng" or "Winter Cherry". The literal meaning of the word "Ashwagandha" is "smell of horse". The fresh roots of the herb emit the smell of horse and there is a commonly held belief that a person consuming the herb may develop the strength and vitality similar to that of a horse [1]. Ashwagandha has been used as a 'Rasayana' and the roots of ashwagandha are regarded as adaptogenic, tonic, aphrodisiac, anti-inflammatory, diuretic, anthelmintic, astringent, thermogenic and stimulant [2]. The ethanol extract obtained from the leaves of field grown ashwagandha has been reported for antioxidant potential [3]. The extract obtained from roots has also been found to have free radicals scavenging activity [4]. The anti-microbial activity of ashwagandha has been reported against many pathogenic bacterial strains [5].

The major phytochemical constituents reported from ashwagandha are alkaloids (isopelletierine, anaferine, cuscohygrine, anahygrine, tropine, somnine, sominiferine, withanine etc), steroidal lactones (withanolides, withaferins), saponins containing an additional acyl group (sitoindoside VII and VIII), withanolides with a glucose at carbon 27 also known as glycol-withanolides (sitoindoside IX and X) and amino acids like aspartic acid, proline, tyrosine, alanine, glycine etc [6]. Many herbal supplements are commercially available containing 1-3\% alkaloids and small amount of withaferine. The pharmacological activity of such products has been claimed as due to presence of alkaloids and/or withaferine. The European Food Safety Authority (EFSA) has categorized some of the alkaloids of ashwagandha as toxic and harmful [7]. These include anaferine, anahygrine, withanine, sominiferine, somnine, tropine etc. Apart from alkaloids, withaferine A has also been categorized as cytotoxic lactone by EFSA [7]. The aim of the present study, therefore, was to assess the toxicity of a purified ashwagandha extract as per OECD (Organisation for Economic Co-operation and Development) guidelines to support its safety for human use.

\section{MATERIALS AND METHODS}

\section{Chemicals and animals}

Purified ashwagandha extract (PAE) manufactured by Arjuna Natural Ltd., Aluva, Kerala, India was used as a test sample for toxicity study. The extract is standardized to contain $35 \%$ glycowithanolides and less than $1 \%$ alkaloids. Reference standards for glycowithanolides (Withanoside IV and Withanoside V) and aglycowithanolides (Withaferin A, Withanolide A and 12-Deoxy-withastramonolide) were purchased from Chromadex, CA, USA. Eight weeks old Sprague Dawley rats weighing 160-180 g were maintained at $24 \pm 2{ }^{\circ} \mathrm{C}$ with $55-70 \%$ relative humidity and lighting was controlled to give $12 \mathrm{~h}$ artificial light ( $6 \mathrm{am}-6 \mathrm{pm})$ each day. Filtered drinking water (Aquaguard) and pellet feed manufactured by M/s. Kerala feeds, Thrissur, India was provided ad libitum. The study was approved by Institutional Animals Ethics Committee of Arjuna Natural Ltd., Kochi, Kerala, India (1524/PO/RcBi/S/11/CPCSEA).

\section{Phytochemical screening of the extract}

The PAE was subjected to qualitative chemical tests for the presence of various categories of phytoconstituents. Quantification of phenolics [8], flavonoids [9], tannins [10], triterpenoids [11], alkaloids [12], saponins [13], carbohydrates and protein [14] was done by existing standard methods.

Quantification of withanolides was done by high performance liquid chromatography (HPLC). The PAE was accurately weighed (125 mg) and transferred into a $25 \mathrm{ml}$ standard flask and made up to a $25 \mathrm{ml}$ 
solution with 50\% aqueous methanol. Standard solutions were prepared by weighing accurately $5 \mathrm{mg}$ standard into a $5 \mathrm{ml}$ standard flask and made up to a $5 \mathrm{ml}$ with methanol. From these stock solutions, $200 \mu \mathrm{l}$ was transferred into $10 \mathrm{ml}$ standard flask and made up to $10 \mathrm{ml}$ with methanol. All the solutions were filtered through $0.2 \mu \mathrm{m}$ filter before injection. HPLC was performed on reverse phase C18 column ( $250 \times 4.6 \mathrm{~mm}$; Shimadzu Co., Japan) using $0.01 \mathrm{M}$ anhydrous potassium dihydrogen orthophosphate (mobile phase A) and acetonitrile (mobile phase B) as the mobile phase and UV detection at $227 \mathrm{~nm}$. The flow rate was $1 \mathrm{ml} / \mathrm{min}$ and run time was $40 \mathrm{~min}$. The concentration gradient for mobile phase B was 5-45\% (18 min), 45-80\% (7 min), hold $80 \%$ (3 $\mathrm{min}$ ), 80-5\% (2 min), hold 5\% (10 min).

\section{Acute toxicity study in rats}

This study was performed in accordance with the OECD guideline for the testing of chemicals, "Acute Oral Toxicity Study (Acute Toxic Class Method)", Guideline No. 423, adopted on December 17, 2001. Six female rats weighing 160-180 g were used in this study. The study was divided into two steps and the animals were acclimatized for $7 \mathrm{~d}$ before the commencement of each step. The animals were fasted overnight before and four hours after dosing. Taking three rats in the first step, a limit test was performed. The test substance (PAE dissolved in distilled water) was administered orally at the dose level of $2000 \mathrm{mg} / \mathrm{kg}$ body weight (bw) with the help of a stainless steel cannula attached with syringe in the first step. No mortality or toxic signs and symptoms were observed in any of the animals at the first step, hence to confirm the findings of first step the next step was performed by taking three more female rats, which were administered the same dose of $2000 \mathrm{mg} / \mathrm{kg} \mathrm{bw}$.

The treated rats were observed for clinical signs of abnormality/mortality five times on day 1 (day of administration) i.e., at $30 \mathrm{~min}$ and four times at hourly (post-administration) intervals and thereafter once daily for a total of $14 \mathrm{~d}$. The body weights of rats were recorded on day 1 , day 7 and day 14 . No mortality or treatment related toxic signs and symptoms were observed in the animals at both the steps. As no toxic signs were noted, no further testing was required. After $14 \mathrm{~d}$, animals were sacrificed and gross pathological changes were recorded.

\section{Sub-chronic (90 d repeated dose) toxicity study}

This study was conducted as per OECD guidelines for testing of chemicals (No.408). One hundred rats (50 males/50 females) were divided into four groups of 20 animals ( 10 males and 10 females) in each and two groups (satellite/recovery groups) of 10 animals (5 males and 5 females) in each group. The animals were acclimatized for seven days before the commencement of dosing. Three groups of 20 rats each (10 male and 10 female) were administered with PAE orally at the dosage levels of $100 \mathrm{mg} / \mathrm{kg}$ bw (low dose; LD), 500 $\mathrm{mg} / \mathrm{kg}$ bw (medium dose; MD) and $1000 \mathrm{mg} / \mathrm{kg}$ bw (high dose; HD) respectively for seven days a week for $90 \mathrm{~d}$ with the help of cannula attached with the syringe. Similarly, fourth group of 20 rats (10 male and 10 female rats) were orally administered with distilled water only (vehicle) for $90 \mathrm{~d}$ and was designated as control group.

Two additional satellite (recovery) groups of 10 rats ( 5 male and 5 female) each were also kept and designated as 'Satellite control' and 'Satellite high dose' and were administered with distilled water (vehicle) and test substance (PAE, $1000 \mathrm{mg} / \mathrm{kg} \mathrm{bw}$ ) respectively daily for $90 \mathrm{~d}$. After terminal sacrifice of the test and control group animals, both recovery group animals (satellite control and satellite high dose) were kept under observation for an additional one month, to check the reversibility, persistence or delayed toxic effect, if any. The animals were observed daily for behavior, appearance and toxicological signs and symptoms. Blood was collected from retro orbital sinus from all the animals before terminal sacrifice for detailed hematological and biochemical evaluation. Criteria used to evaluate compound related effects included; appearance, behavior, morbidity, mortality, body weights, feed consumption, hematological and biochemical analysis, organ weights, necropsy and histopathology.

\section{Statistical analysis}

Using Graph Pad Prism software the data was analysed by one-way analyses of variance (ANOVA). Following ANOVA, Dunnett's pairwise comparison of means of treated groups with control group mean was carried out individually. The data is presented as the mean \pm SEM (standard error of mean) and $p$ value $<0.05$ was considered as significant.

\section{RESULTS}

\section{Phytochemical screening}

The PAE was found to be rich in triterpinoids, saponins and carbohydrates. The percentage of withanolide glycosides (glycowithanolides) was 35\% whereas the percentage of alkaloids in PAE was below $1 \%$.

\section{Acute toxicity study in rats}

No clinical sign of toxicity was observed in any of the treated rat at the dose level of $2000 \mathrm{mg} / \mathrm{kg}$ bw. No mortality was observed in the animals at step I and step II administered with the PAE at 2000 $\mathrm{mg} / \mathrm{kg}$ bw. Individual body weights were recorded prior to oral administration (day 1) and on days 7 and 14 following oral administration. The gain in body weights was normal in all the rats (table 1). All animals were sacrificed at the end of the study and they did not reveal any abnormality of pathological significance. External examination of terminally sacrificed rats also did not reveal any abnormality of pathological significance. Based on these findings of the acute oral toxicity (Acute Toxic Class Method) of the PAE in rats, the $\mathrm{LD}_{50}$ of the extract may be classified as GHS (Globally Harmonized System) category $5\left(L_{50}>2000 \mathrm{mg} / \mathrm{kg} \mathrm{bw}\right.$ ) as per OECD Guideline No. 423, December 2001.

Table 1: Body weight, body weight changes and pre-terminal deaths in acute toxicity study

\begin{tabular}{llllllll}
\hline Dose (mg/kg b.w.) & Rat No. & Sex & \multicolumn{2}{c}{ Body weight (g) } & & \\
\cline { 4 - 7 } & & & Day 1 & Day 7 & Day 7-Day 1 & Day 14 & Day 14-Day 1 \\
\hline 2000 & 1 & Female & 161 & 172 & 11 & 17 & 17 \\
& 2 & Female & 164 & 170 & 6 & 176 & 12 \\
& 3 & Female & 160 & 167 & 7 & 173 & 13 \\
& 4 & Female & 159 & 169 & 10 & 174 & 15 \\
& 5 & Female & 178 & 184 & 6 & 190 & 12 \\
& 6 & Female & 172 & 177 & 5 & 191 & 19 \\
\hline
\end{tabular}

\section{Sub-chronic toxicity study}

No treatment related toxic sign and symptoms were observed in LD, MD and HD group animals when compared to their respective control counter parts. Body weight gain and feed consumption of all the treatment group animals was comparable to their control counterparts (data not shown).

There were no variations in the hematological parameters of animals of LD, MD and HD groups when compared to the control group animals (table 2-3). Similarly, the biochemical parameters of animals of all the treatment groups i.e. LD, MD and HD were comparable to the biochemical parameters of the control group animals at the terminal sacrifice (table 4-5).

None of the animals died during the study in any of the treated groups as well as the control group. After completion of dosing period of $90 \mathrm{~d}(120 \mathrm{~d}$ for recovery groups) all the animals (treatment and control) were sacrificed and were examined for 
gross pathological findings. Organ weights of animals of all treatment groups were comparable to their respective control counter parts (data not shown). There were no significant histopathological changes in the animals of LD, MD and HD group when compared to their control counterparts (fig. 1).
In this study, the repeated administration of PAE for $90 \mathrm{~d}$, by oral route, to rats at the highest dosage level of $1000 \mathrm{mg} / \mathrm{kg} \mathrm{bw}$ did not induce any observable toxic effects, when compared to its corresponding control group of animals. Therefore, this dose may be considered as the No Observed Adverse Effect Level (NOAEL).

Table 2: Heamatological parameters of male rats in sub-chronic ( $90 \mathrm{~d}$ repeated dose) toxicity study

\begin{tabular}{|c|c|c|c|c|c|c|c|c|c|c|c|c|}
\hline Treatment & $\begin{array}{l}\text { WBC (X } \\
\left.10^{3}\right)\end{array}$ & $\begin{array}{l}\text { RBC } \\
(X \\
\left.10^{6}\right)\end{array}$ & $\begin{array}{l}\text { Hb } \\
\text { (gm/ } \\
\text { dl) }\end{array}$ & $\mathrm{N} \%$ & E\% & L\% & PCV & MCV & MCH & MCHC & $\begin{array}{l}\text { Platelet } \\
\text { count }(X \\
\left.10^{5}\right)\end{array}$ & $\begin{array}{l}\text { RDW } \\
\text { (CV) }\end{array}$ \\
\hline $\begin{array}{l}\text { Control (0 } \\
\mathrm{mg} / \mathrm{kg} \mathrm{bw})\end{array}$ & $6.13 \pm 0.45$ & $\begin{array}{l}7.14 \pm \\
0.77\end{array}$ & $\begin{array}{l}15.43 \\
\pm 1.21\end{array}$ & $\begin{array}{l}26.00 \\
\pm 2.51\end{array}$ & $\begin{array}{l}4.67 \pm 0 . \\
28\end{array}$ & $\begin{array}{l}69.33 \pm 5 \\
.21\end{array}$ & $\begin{array}{l}47.93 \pm 4 \\
.22\end{array}$ & $\begin{array}{l}58.90 \pm 4 \\
.36\end{array}$ & $\begin{array}{l}17.73 \pm 1 \\
.63\end{array}$ & $\begin{array}{l}30.13 \\
\pm 2.19\end{array}$ & $8.04 \pm 0.74$ & $\begin{array}{l}18.40 \pm 1 \\
.42\end{array}$ \\
\hline $\begin{array}{l}\text { Low dose } \\
(100 \mathrm{mg} / \mathrm{kg} \\
\text { bw) }\end{array}$ & $7.14 \pm 0.45$ & $\begin{array}{l}8.09 \pm \\
0.95\end{array}$ & $\begin{array}{l}14.63 \\
\pm 1.32\end{array}$ & $\begin{array}{l}23.00 \\
\pm 1.96\end{array}$ & $\begin{array}{l}5.00 \pm 0 . \\
34\end{array}$ & $\begin{array}{l}72.00 \pm 5 \\
.66\end{array}$ & $\begin{array}{l}52.23 \pm 4 \\
.64\end{array}$ & $\begin{array}{l}57.53 \pm 5 \\
.64\end{array}$ & $\begin{array}{l}17.23 \pm 1 \\
.88\end{array}$ & $\begin{array}{l}29.93 \\
\pm 2.41\end{array}$ & $9.58 \pm 0.88$ & $\begin{array}{l}18.80 \pm 1 \\
.11\end{array}$ \\
\hline $\begin{array}{l}\text { Medium } \\
\text { dose (500 } \\
\mathrm{mg} / \mathrm{kg} \mathrm{bw})\end{array}$ & $7.39 \pm 0.42$ & $\begin{array}{l}8.12 \pm \\
0.75\end{array}$ & $\begin{array}{l}14.53 \\
\pm 0.98\end{array}$ & $\begin{array}{l}24.67 \\
\pm 3.65\end{array}$ & $\begin{array}{l}4.33 \pm 0 . \\
21\end{array}$ & $\begin{array}{l}71.00 \pm 4 \\
.57\end{array}$ & $\begin{array}{l}45.70 \pm 3 \\
.66\end{array}$ & $\begin{array}{l}58.90 \pm 6 \\
.41\end{array}$ & $\begin{array}{l}17.43 \pm 1 \\
.41\end{array}$ & $\begin{array}{l}29.57 \\
\pm 2.32\end{array}$ & $7.84 \pm 0.45$ & $\begin{array}{l}17.13 \pm 1 \\
.18\end{array}$ \\
\hline $\begin{array}{l}\text { High dose } \\
(1000 \\
\text { mg/kg bw) }\end{array}$ & $7.28 \pm 0.68$ & $\begin{array}{l}8.42 \pm \\
0.86\end{array}$ & $\begin{array}{l}15.27 \\
\pm 0.94\end{array}$ & $\begin{array}{l}25.33 \\
\pm 3.14\end{array}$ & $\begin{array}{l}3.67 \pm 0 \\
18\end{array}$ & $\begin{array}{l}71.00 \pm 5 \\
.33\end{array}$ & $\begin{array}{l}47.87 \pm 5 \\
.22\end{array}$ & $\begin{array}{l}55.03 \pm 5 \\
.22\end{array}$ & $\begin{array}{l}16.40 \pm 1 \\
.08\end{array}$ & $\begin{array}{l}29.80 \\
\pm 2.17\end{array}$ & $8.14 \pm 0.58$ & $\begin{array}{l}19.17 \pm 0 \\
.99\end{array}$ \\
\hline $\begin{array}{l}\text { Control } \\
\text { recovery ( } 0 \\
\mathrm{mg} / \mathrm{kg} \mathrm{bw} \text { ) }\end{array}$ & $8.35 \pm 0.59$ & $\begin{array}{l}8.48 \pm \\
0.96\end{array}$ & $\begin{array}{l}14.53 \\
\pm 1.05\end{array}$ & $\begin{array}{l}24.00 \\
\pm 2.58\end{array}$ & $\begin{array}{l}3.67 \pm 0 . \\
26\end{array}$ & $\begin{array}{l}72.33 \pm 6 \\
.32\end{array}$ & $\begin{array}{l}42.40 \pm 4 \\
.17\end{array}$ & $\begin{array}{l}51.17 \pm 4 \\
.85\end{array}$ & $\begin{array}{l}16.37 \pm 1 \\
.41\end{array}$ & $\begin{array}{l}31.90 \\
\pm 2.09\end{array}$ & $\begin{array}{l}10.43 \pm 1.2 \\
1\end{array}$ & $\begin{array}{l}18.27 \pm 1 \\
.84\end{array}$ \\
\hline $\begin{array}{l}\text { High dose } \\
\text { recovery } \\
(1000 \\
\mathrm{mg} / \mathrm{kg} \mathrm{bw})\end{array}$ & $6.95 \pm 0.37$ & $\begin{array}{l}8.16 \pm \\
0.81\end{array}$ & $\begin{array}{l}14.47 \\
\pm 1.17\end{array}$ & $\begin{array}{l}22.67 \\
\pm 2.67\end{array}$ & $\begin{array}{l}4.00 \pm 0 . \\
14\end{array}$ & $\begin{array}{l}73.33 \pm 6 \\
.78\end{array}$ & $\begin{array}{l}46.37 \pm 4 \\
.22\end{array}$ & $\begin{array}{l}52.40 \pm 4 \\
.06\end{array}$ & $\begin{array}{l}16.80 \pm 1 \\
.11\end{array}$ & $\begin{array}{l}32.07 \\
\pm 3.11\end{array}$ & $9.44 \pm 0.69$ & $\begin{array}{l}18.77 \pm 1 \\
.92\end{array}$ \\
\hline
\end{tabular}

Analysis of variance (ANOVA) was used for statistical analysis. Data are presented as the mean \pm SEM. $P>0.05$, compared with the respective control. No significant differences were observed between the test and control groups. Hb=Hemoglobin; N=Neutrophils; E=Eosinophils; L=Lymphocytes; PCV=Packed Cell Volume (Hematocrit); MCV= Mean Corpuscular Volume; MCH= Mean Corpuscular Hemoglobin; MCHC= Mean Corpuscular Hemoglobin Concentration; RDW= Red Blood Cell Distribution Width.

Table 3: Heamatological parameters of female rats in sub-chronic ( $90 \mathrm{~d}$ repeated dose) toxicity study

\begin{tabular}{|c|c|c|c|c|c|c|c|c|c|c|c|c|}
\hline Treatment & $\begin{array}{l}\text { WBC } \\
\left(X 10^{3}\right)\end{array}$ & $\begin{array}{l}\text { RBC } \\
\left(X 10^{6}\right)\end{array}$ & $\begin{array}{l}\text { Hb } \\
\text { (gm/dl) }\end{array}$ & N\% & E\% & L\% & PCV & MCV & MCH & MCHC & $\begin{array}{l}\text { Platelet } \\
\text { count } \\
(\mathrm{X} \mathrm{105}) \\
\end{array}$ & $\begin{array}{l}\text { RDW } \\
\text { (CV) }\end{array}$ \\
\hline $\begin{array}{l}\text { Control (0 } \\
\mathrm{mg} / \mathrm{kg} \text { bw) }\end{array}$ & $\begin{array}{l}7.48 \pm 0 \\
.48\end{array}$ & $\begin{array}{l}6.96 \pm 0 . \\
81\end{array}$ & $\begin{array}{l}13.30 \pm \\
0.96\end{array}$ & $\begin{array}{l}20.67 \\
\pm 1.54\end{array}$ & $\begin{array}{l}3.67 \pm 0 . \\
31\end{array}$ & $\begin{array}{l}75.67 \pm 4 \\
51\end{array}$ & $\begin{array}{l}41.57 \pm 3 . \\
25\end{array}$ & $\begin{array}{l}57.40 \pm 3 . \\
41\end{array}$ & $\begin{array}{l}16.97 \pm 1 \\
14\end{array}$ & $\begin{array}{l}29.60 \pm 2 . \\
14\end{array}$ & $\begin{array}{l}8.67 \pm 0 . \\
58\end{array}$ & $\begin{array}{l}15.27 \pm 1 \\
.11\end{array}$ \\
\hline $\begin{array}{l}\text { Low dose } \\
(100 \\
\mathrm{mg} / \mathrm{kg} \text { bw })\end{array}$ & $\begin{array}{l}7.08 \pm 0 \\
.41\end{array}$ & $\begin{array}{l}7.97 \pm 0 . \\
74\end{array}$ & $\begin{array}{l}14.50 \pm \\
1.14\end{array}$ & $\begin{array}{l}20.33 \\
\pm 2.14\end{array}$ & $\begin{array}{l}3.67 \pm 0 . \\
14\end{array}$ & $\begin{array}{l}76.00 \pm 4 \\
62\end{array}$ & $\begin{array}{l}49.47 \pm 3 \\
41\end{array}$ & $\begin{array}{l}62.03 \pm 4 \\
96\end{array}$ & $\begin{array}{l}18.20 \pm 1 . \\
36\end{array}$ & $\begin{array}{l}29.30 \pm 2 . \\
19\end{array}$ & $\begin{array}{l}8.25 \pm 0 . \\
93\end{array}$ & $\begin{array}{l}16.20 \pm 1 \\
.23\end{array}$ \\
\hline $\begin{array}{l}\text { Medium } \\
\text { dose ( } 500 \\
\mathrm{mg} / \mathrm{kg} \mathrm{bw})\end{array}$ & $\begin{array}{l}6.87 \pm 0 \\
.36\end{array}$ & $\begin{array}{l}8.44 \pm 0 . \\
76\end{array}$ & $\begin{array}{l}12.60 \pm \\
1.16\end{array}$ & $\begin{array}{l}23.00 \\
\pm 2.65\end{array}$ & $\begin{array}{l}3.67 \pm 0 . \\
36\end{array}$ & $\begin{array}{l}73.33 \pm 4 \\
19\end{array}$ & $\begin{array}{l}45.33 \pm 2 . \\
58\end{array}$ & $\begin{array}{l}60.90 \pm 4 \\
55\end{array}$ & $\begin{array}{l}17.60 \pm 1 . \\
58\end{array}$ & $\begin{array}{l}28.90 \pm 3 \\
15\end{array}$ & $\begin{array}{l}8.48 \pm 0 . \\
44\end{array}$ & $\begin{array}{l}16.47 \pm 0 \\
.95\end{array}$ \\
\hline $\begin{array}{l}\text { High dose } \\
(1000 \\
\mathrm{mg} / \mathrm{kg} \mathrm{bw})\end{array}$ & $\begin{array}{l}6.87 \pm 0 \\
.65\end{array}$ & $\begin{array}{l}7.06 \pm 0 . \\
77\end{array}$ & $\begin{array}{l}12.17 \pm \\
1.11\end{array}$ & $\begin{array}{l}20.33 \\
\pm 2.17\end{array}$ & $\begin{array}{l}3.00 \pm 0 . \\
41\end{array}$ & $\begin{array}{l}76.67 \pm 5 \\
68\end{array}$ & $\begin{array}{l}41.17 \pm 3 \\
65\end{array}$ & $\begin{array}{l}58.13 \pm 4 \\
17\end{array}$ & $\begin{array}{l}17.20 \pm 1 \\
98\end{array}$ & $\begin{array}{l}29.60 \pm 2 . \\
97\end{array}$ & $\begin{array}{l}7.47 \pm 0 . \\
35\end{array}$ & $\begin{array}{l}14.50 \pm 0 \\
.88\end{array}$ \\
\hline $\begin{array}{l}\text { Control } \\
\text { recovery ( } 0 \\
\mathrm{mg} / \mathrm{kg} \text { bw) }\end{array}$ & $\begin{array}{l}6.90 \pm 0 \\
.58\end{array}$ & $\begin{array}{l}7.56 \pm 0 . \\
85\end{array}$ & $\begin{array}{l}13.30 \pm \\
1.18\end{array}$ & $\begin{array}{l}20.33 \\
\pm 2.84\end{array}$ & $\begin{array}{l}3.00 \pm 0 . \\
31\end{array}$ & $\begin{array}{l}76.67 \pm 5 . \\
44\end{array}$ & $\begin{array}{l}40.93 \pm 4 \\
14\end{array}$ & $\begin{array}{l}54.13 \pm 3 \\
68\end{array}$ & $\begin{array}{l}17.60 \pm 2 . \\
17\end{array}$ & $\begin{array}{l}32.50 \pm 2 \\
77\end{array}$ & $\begin{array}{l}8.93 \pm 0 \\
74\end{array}$ & $\begin{array}{l}15.47 \pm 1 \\
.24\end{array}$ \\
\hline $\begin{array}{l}\text { High dose } \\
\text { recovery } \\
(1000 \\
\mathrm{mg} / \mathrm{kg} \mathrm{bw})\end{array}$ & $\begin{array}{l}6.61 \pm 0 \\
.63\end{array}$ & $\begin{array}{l}7.34 \pm 0 . \\
82\end{array}$ & $\begin{array}{l}13.17 \pm \\
0.97\end{array}$ & $\begin{array}{l}22.00 \\
\pm 1.59\end{array}$ & $\begin{array}{l}3.33 \pm 0 . \\
19\end{array}$ & $\begin{array}{l}74.67 \pm 7 \\
25\end{array}$ & $\begin{array}{l}42.33 \pm 3 \\
87\end{array}$ & $\begin{array}{l}57.77 \pm 3 \\
47\end{array}$ & $\begin{array}{l}17.93 \pm 1 \\
41\end{array}$ & $\begin{array}{l}31.10 \pm 3 \\
24\end{array}$ & $\begin{array}{l}9.17 \pm 1 \\
04\end{array}$ & $\begin{array}{l}15.13 \pm 1 \\
.05\end{array}$ \\
\hline
\end{tabular}

Analysis of variance (ANOVA) was used for statistical analysis. Data are presented as the mean \pm SEM. P $>0.05$, compared with the respective control. No significant differences were observed between the test and control groups. Hb=Hemoglobin; N=Neutrophils; E=Eosinophils; L=Lymphocytes; PCV=Packed Cell Volume (Hematocrit); MCV= Mean Corpuscular Volume; MCH= Mean Corpuscular Hemoglobin; MCHC= Mean Corpuscular Hemoglobin Concentration; RDW= Red Blood Cell Distribution Width

Table 4: Biochemical parameters of male rats in in sub-chronic ( $90 \mathrm{~d}$ repeated dose) toxicity study

\begin{tabular}{|c|c|c|c|c|c|c|c|c|c|c|c|c|}
\hline Treatment & $\begin{array}{l}\text { CHO } \\
\text { (mg/dl) }\end{array}$ & $\begin{array}{l}\text { TG } \\
\text { (mg/dl) }\end{array}$ & $\begin{array}{l}\text { ALKP } \\
\text { (U/I) }\end{array}$ & $\begin{array}{l}\text { T. Bil } \\
\text { (mg/dl) }\end{array}$ & $\begin{array}{l}\text { T. Pro } \\
\text { (g/dl) }\end{array}$ & $\begin{array}{l}\text { Alb } \\
\text { (g/dl) }\end{array}$ & $\begin{array}{l}\text { Glo } \\
\text { (g/dl) }\end{array}$ & $\begin{array}{l}\text { SGOT } \\
\text { (U/I) }\end{array}$ & $\begin{array}{l}\text { SGPT } \\
\text { (U/I) }\end{array}$ & $\begin{array}{l}\text { Urea } \\
\text { (mg/dl) }\end{array}$ & $\begin{array}{l}\text { Cre } \\
\text { (mg/dl) }\end{array}$ & $\begin{array}{l}\text { FBS } \\
\text { (mg/dl) }\end{array}$ \\
\hline $\begin{array}{l}\text { Control (0 } \\
\mathrm{mg} / \mathrm{kg} \mathrm{bw})\end{array}$ & $\begin{array}{l}62.67 \pm \\
2.54\end{array}$ & $\begin{array}{l}51.67 \pm \\
2.58\end{array}$ & $\begin{array}{l}1.00 \pm 0 . \\
75\end{array}$ & $\begin{array}{l}0.10 \pm 0 . \\
01\end{array}$ & $\begin{array}{l}6.57 \pm 0 . \\
39\end{array}$ & $\begin{array}{l}3.93 \pm 0 . \\
19\end{array}$ & $\begin{array}{l}2.63 \pm 0 . \\
18\end{array}$ & $\begin{array}{l}87.00 \pm 4 . \\
22\end{array}$ & $\begin{array}{l}42.67 \pm 2 . \\
58\end{array}$ & $\begin{array}{l}29.67 \pm 1 \\
84\end{array}$ & $\begin{array}{l}0.29 \pm 0 . \\
02\end{array}$ & $\begin{array}{l}89.00 \pm 6 . \\
35\end{array}$ \\
\hline $\begin{array}{l}\text { Low dose } \\
(100 \\
\mathrm{mg} / \mathrm{kg} \mathrm{bw})\end{array}$ & $\begin{array}{l}65.67 \pm \\
2.88\end{array}$ & $\begin{array}{l}55.00 \pm \\
3.21\end{array}$ & $\begin{array}{l}1.00 \pm 0 \\
74\end{array}$ & $\begin{array}{l}0.10 \pm 0 . \\
01\end{array}$ & $\begin{array}{l}6.60 \pm 0 \\
32\end{array}$ & $\begin{array}{l}3.87 \pm 0 \\
25\end{array}$ & $\begin{array}{l}2.73 \pm 0 . \\
24\end{array}$ & $\begin{array}{l}82.33 \pm 5 \\
35\end{array}$ & $\begin{array}{l}40.00 \pm 3 \\
14\end{array}$ & $\begin{array}{l}36.67 \pm 1 \\
96\end{array}$ & $\begin{array}{l}0.36 \pm 0 \\
03\end{array}$ & $\begin{array}{l}90.33 \pm 7 \\
14\end{array}$ \\
\hline
\end{tabular}




\begin{tabular}{|c|c|c|c|c|c|c|c|c|c|c|c|c|}
\hline $\begin{array}{l}\text { Medium } \\
\text { dose }(500 \\
\mathrm{mg} / \mathrm{kg} \mathrm{bw})\end{array}$ & $\begin{array}{l}59.00 \pm \\
2.47\end{array}$ & $\begin{array}{l}52.00 \pm \\
2.24\end{array}$ & $\begin{array}{l}1.00 \pm 0 . \\
71\end{array}$ & $\begin{array}{l}0.10 \pm 0 . \\
01\end{array}$ & $\begin{array}{l}6.43 \pm 0 \\
36\end{array}$ & $\begin{array}{l}3.93 \pm 0 . \\
22\end{array}$ & $\begin{array}{l}2.50 \pm 0 . \\
22\end{array}$ & $\begin{array}{l}88.33 \pm 6 . \\
21\end{array}$ & $\begin{array}{l}43.00 \pm 2 . \\
54\end{array}$ & $\begin{array}{l}37.00 \pm 2 \\
14\end{array}$ & $\begin{array}{l}0.33 \pm 0 \\
03\end{array}$ & $\begin{array}{l}91.33 \pm 7 \\
52\end{array}$ \\
\hline $\begin{array}{l}\text { High dose } \\
(1000 \\
\mathrm{mg} / \mathrm{kg} \mathrm{bw})\end{array}$ & $\begin{array}{l}65.67 \pm \\
2.65\end{array}$ & $\begin{array}{l}54.67 \pm \\
2.51\end{array}$ & $\begin{array}{l}1.33 \pm 0 . \\
36\end{array}$ & $\begin{array}{l}0.10 \pm 0 \\
01\end{array}$ & $\begin{array}{l}6.77 \pm 0 \\
45\end{array}$ & $\begin{array}{l}3.73 \pm 0 . \\
21\end{array}$ & $\begin{array}{l}3.03 \pm 0 \\
23\end{array}$ & $\begin{array}{l}87.67 \pm 5 \\
87\end{array}$ & $\begin{array}{l}46.33 \pm 2 \\
63\end{array}$ & $\begin{array}{l}42.00 \pm 2 \\
65\end{array}$ & $\begin{array}{l}0.33 \pm 0 \\
02\end{array}$ & $\begin{array}{l}86.33 \pm 6 . \\
24\end{array}$ \\
\hline $\begin{array}{l}\text { Control } \\
\text { recovery (0 } \\
\mathrm{mg} / \mathrm{kg} \mathrm{bw} \text { ) }\end{array}$ & $\begin{array}{l}67.67 \pm \\
2.74\end{array}$ & $\begin{array}{l}59.00 \pm \\
2.48\end{array}$ & $\begin{array}{l}1.00 \pm 0 . \\
74\end{array}$ & $\begin{array}{l}0.10 \pm 0 . \\
01\end{array}$ & $\begin{array}{l}7.13 \pm 0 . \\
57\end{array}$ & $\begin{array}{l}3.73 \pm 0 . \\
23\end{array}$ & $\begin{array}{l}3.40 \pm 0 . \\
29\end{array}$ & $\begin{array}{l}81.67 \pm 6 \\
71\end{array}$ & $\begin{array}{l}42.67 \pm 3 \\
14\end{array}$ & $\begin{array}{l}34.00 \pm 3 \\
02\end{array}$ & $\begin{array}{l}0.25 \pm 0 \\
03\end{array}$ & $\begin{array}{l}92.67 \pm 7 \\
84\end{array}$ \\
\hline $\begin{array}{l}\text { High dose } \\
\text { recovery } \\
(1000 \\
\mathrm{mg} / \mathrm{kg} \mathrm{bw})\end{array}$ & $\begin{array}{l}66.67 \pm \\
3.21\end{array}$ & $\begin{array}{l}58.00 \pm \\
2.63\end{array}$ & $\begin{array}{l}1.33 \pm 0 . \\
73\end{array}$ & $\begin{array}{l}0.10 \pm 0 . \\
01\end{array}$ & $\begin{array}{l}7.17 \pm 0 . \\
39\end{array}$ & $\begin{array}{l}3.57 \pm 0 \\
29\end{array}$ & $\begin{array}{l}3.60 \pm 0 . \\
38\end{array}$ & $\begin{array}{l}82.00 \pm 6 \\
33\end{array}$ & $\begin{array}{l}40.33 \pm 3 \\
1\end{array}$ & $\begin{array}{l}41.33 \pm 4 \\
05\end{array}$ & $\begin{array}{l}0.37 \pm 0 \\
02\end{array}$ & $\begin{array}{l}88.00 \pm 6 . \\
66\end{array}$ \\
\hline
\end{tabular}

Analysis of variance (ANOVA) was used for statistical analysis. Data are presented as the mean \pm SEM. P $>0.05$, compared with the respective control. No significant differences were observed between the test and control groups. $\mathrm{CHO}=\mathrm{Cholesterol;} \mathrm{TG}=\mathrm{Triglycerides}$; $\mathrm{ALKP}=\mathrm{Alkaline}$ phosphatase; T. Bil=Total Bilirubin; T. Pro=Total Protein; Alb=Albumin; Glo=Globulin; SGOT=Serum glutamic oxaloacetic transaminase; SGPT=Serum glutamic pyruvic transaminase; Cre=Creatinine; FBS=Fasting Blood Sugar

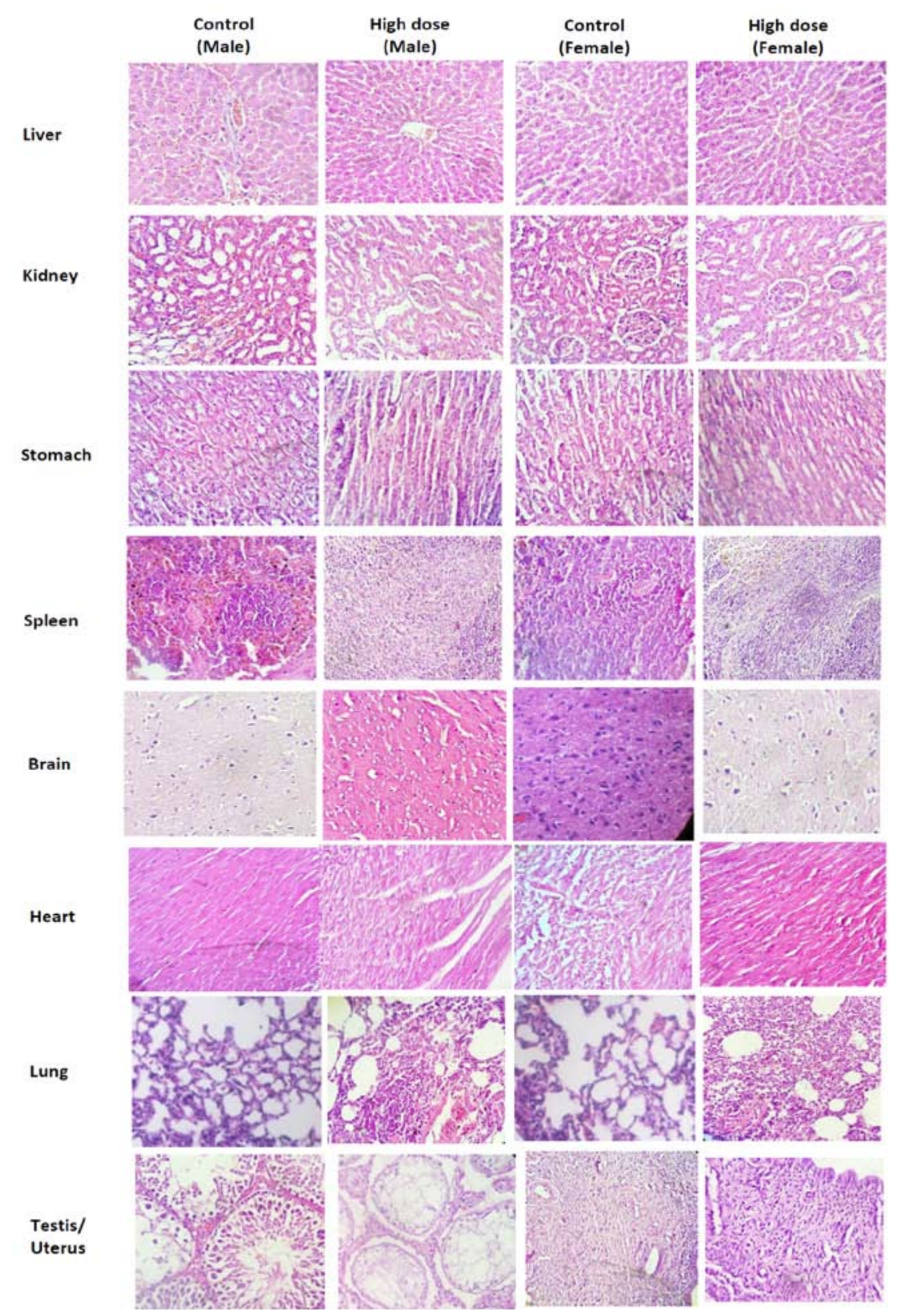

Fig. 1: Histopathological sections of major organs in $90 \mathrm{~d}$ repeated dose toxicity study

Table 5: Biochemical parameters of female rats in in sub-chronic ( $90 \mathrm{~d}$ repeated dose) toxicity study 


\begin{tabular}{|c|c|c|c|c|c|c|c|c|c|c|c|c|}
\hline Treatment & $\begin{array}{l}\text { CHO } \\
\text { (mg/dl) }\end{array}$ & $\begin{array}{l}\text { TG } \\
\text { (mg/dl) }\end{array}$ & $\begin{array}{l}\text { ALKP } \\
(\mathrm{U} / \mathrm{I})\end{array}$ & $\begin{array}{l}\text { T. Bil } \\
\text { (mg/dl) }\end{array}$ & $\begin{array}{l}\text { T. Pro } \\
\text { (g/dl) }\end{array}$ & $\begin{array}{l}\text { Alb } \\
(\mathrm{g} / \mathrm{dl})\end{array}$ & $\begin{array}{l}\text { Glo } \\
\text { (g/dl) }\end{array}$ & $\begin{array}{l}\text { SGOT } \\
\text { (U/I) }\end{array}$ & $\begin{array}{l}\text { SGPT } \\
\text { (U/I) }\end{array}$ & $\begin{array}{l}\text { Urea } \\
\text { (mg/dl) }\end{array}$ & $\begin{array}{l}\text { Cre } \\
\text { (mg/dl) }\end{array}$ & $\begin{array}{l}\text { FBS } \\
\text { (mg/dl) }\end{array}$ \\
\hline $\begin{array}{l}\text { Control (0 } \\
\mathrm{mg} / \mathrm{kg} \mathrm{bw})\end{array}$ & $\begin{array}{l}65.33 \pm \\
2.67\end{array}$ & $\begin{array}{l}55.67 \pm \\
2.15\end{array}$ & $\begin{array}{l}1.00 \pm 0 . \\
33\end{array}$ & $\begin{array}{l}0.10 \pm 0 . \\
01\end{array}$ & $\begin{array}{l}6.90 \pm 0 . \\
41\end{array}$ & $\begin{array}{l}3.57 \pm 0 . \\
21\end{array}$ & $\begin{array}{l}3.33 \pm 0 . \\
22\end{array}$ & $\begin{array}{l}92.33 \pm 7 \\
54\end{array}$ & $\begin{array}{l}36.33 \pm 2 . \\
55\end{array}$ & $\begin{array}{l}37.67 \pm 2 . \\
44\end{array}$ & $\begin{array}{l}0.39 \pm 0 . \\
02\end{array}$ & $\begin{array}{l}88.00 \pm 7 . \\
21\end{array}$ \\
\hline $\begin{array}{l}\text { Low dose } \\
(100 \\
\mathrm{mg} / \mathrm{kg} \mathrm{bw})\end{array}$ & $\begin{array}{l}62.67 \pm \\
2.44\end{array}$ & $\begin{array}{l}51.67 \pm \\
2.18\end{array}$ & $\begin{array}{l}1.33 \pm 0 . \\
66\end{array}$ & $\begin{array}{l}0.10 \pm 0 . \\
01\end{array}$ & $\begin{array}{l}6.50 \pm 0 \\
36\end{array}$ & $\begin{array}{l}3.97 \pm 0 . \\
18\end{array}$ & $\begin{array}{l}2.53 \pm 0 . \\
26\end{array}$ & $\begin{array}{l}88.33 \pm 7 \\
66\end{array}$ & $\begin{array}{l}44.33 \pm 2 . \\
36\end{array}$ & $\begin{array}{l}44.00 \pm 2 . \\
54\end{array}$ & $\begin{array}{l}0.43 \pm 0 \\
04\end{array}$ & $\begin{array}{l}89.67 \pm 7 \\
54\end{array}$ \\
\hline $\begin{array}{l}\text { Medium } \\
\text { dose ( } 500 \\
\mathrm{mg} / \mathrm{kg} \mathrm{bw})\end{array}$ & $\begin{array}{l}66.33 \pm \\
2.63\end{array}$ & $\begin{array}{l}57.33 \pm \\
2.34\end{array}$ & $\begin{array}{l}1.33 \pm 0 . \\
71\end{array}$ & $\begin{array}{l}0.10 \pm 0 . \\
01\end{array}$ & $\begin{array}{l}7.00 \pm 0 . \\
64\end{array}$ & $\begin{array}{l}4.13 \pm 0 . \\
29\end{array}$ & $\begin{array}{l}2.87 \pm 0 \\
28\end{array}$ & $\begin{array}{l}88.67 \pm 7 \\
38\end{array}$ & $\begin{array}{l}36.33 \pm 3 \\
21\end{array}$ & $\begin{array}{l}49.67 \pm 2 \\
66\end{array}$ & $\begin{array}{l}0.43 \pm 0 \\
03\end{array}$ & $\begin{array}{l}81.67 \pm 8 \\
65\end{array}$ \\
\hline $\begin{array}{l}\text { High dose } \\
(1000 \\
\mathrm{mg} / \mathrm{kg} \text { bw })\end{array}$ & $\begin{array}{l}68.33 \pm \\
2.61\end{array}$ & $\begin{array}{l}50.33 \pm \\
2.19\end{array}$ & $\begin{array}{l}1.00 \pm 0 . \\
69\end{array}$ & $\begin{array}{l}0.10 \pm 0 \\
01\end{array}$ & $\begin{array}{l}7.03 \pm 0 \\
58\end{array}$ & $\begin{array}{l}3.83 \pm 0 . \\
28\end{array}$ & $\begin{array}{l}3.20 \pm 0 . \\
36\end{array}$ & $\begin{array}{l}91.67 \pm 9 . \\
51\end{array}$ & $\begin{array}{l}38.00 \pm 3 \\
11\end{array}$ & $\begin{array}{l}43.67 \pm 2 \\
67\end{array}$ & $\begin{array}{l}0.42 \pm 0 \\
03\end{array}$ & $\begin{array}{l}89.33 \pm 9 . \\
21\end{array}$ \\
\hline $\begin{array}{l}\text { Control } \\
\text { recovery ( } 0 \\
\mathrm{mg} / \mathrm{kg} \mathrm{bw} \text { ) }\end{array}$ & $\begin{array}{l}61.00 \pm \\
2.18\end{array}$ & $\begin{array}{l}56.67 \pm \\
2.38\end{array}$ & $\begin{array}{l}1.00 \pm 0 . \\
54\end{array}$ & $\begin{array}{l}0.10 \pm 0 . \\
01\end{array}$ & $\begin{array}{l}7.20 \pm 0 . \\
66\end{array}$ & $\begin{array}{l}3.87 \pm 0 . \\
19\end{array}$ & $\begin{array}{l}3.33 \pm 0 . \\
34\end{array}$ & $\begin{array}{l}86.33 \pm 8 \\
87\end{array}$ & $\begin{array}{l}42.67 \pm 2 \\
58\end{array}$ & $\begin{array}{l}35.67 \pm 2 \\
14\end{array}$ & $\begin{array}{l}0.33 \pm 0 \\
01\end{array}$ & $\begin{array}{l}87.33 \pm 5 \\
41\end{array}$ \\
\hline $\begin{array}{l}\text { High dose } \\
\text { recovery } \\
(1000 \\
\mathrm{mg} / \mathrm{kg} \mathrm{bw})\end{array}$ & $\begin{array}{l}63.33 \pm \\
3.22\end{array}$ & $\begin{array}{l}53.67 \pm \\
2.67\end{array}$ & $\begin{array}{l}1.00 \pm 0 . \\
58\end{array}$ & $\begin{array}{l}0.10 \pm 0 . \\
01\end{array}$ & $\begin{array}{l}7.47 \pm 0 . \\
84\end{array}$ & $\begin{array}{l}4.17 \pm 0 . \\
35\end{array}$ & $\begin{array}{l}3.30 \pm 0 . \\
31\end{array}$ & $\begin{array}{l}93.33 \pm 9 \\
28\end{array}$ & $\begin{array}{l}44.33 \pm 3 \\
69\end{array}$ & $\begin{array}{l}36.00 \pm 2 . \\
58\end{array}$ & $\begin{array}{l}0.41 \pm 0 . \\
02\end{array}$ & $\begin{array}{l}88.67 \pm 6 \\
58\end{array}$ \\
\hline
\end{tabular}

Analysis of variance (ANOVA) was used for statistical analysis. Data are presented as the mean \pm SEM. P $>0.05$, compared with the respective control. No significant differences were observed between the test and control groups. $\mathrm{CHO}=\mathrm{Cholesterol}$; $\mathrm{TG}=\mathrm{Triglycerides}$; $\mathrm{ALKP}=\mathrm{Alkaline}$ phosphatase; $\mathrm{T}$. Bil=Total Bilirubin; T. Pro=Total Protein; Alb=Albumin; Glo=Globulin; SGOT=Serum glutamic oxaloacetic transaminase; SGPT=Serum glutamic pyruvic transaminase; Cre=Creatinine; FBS=Fasting Blood Sugar

\section{DISCUSSION}

Ashwagandha has a number of benefits and pharmacological activities. Root powder of ashwagandha has been used in the Ayurvedic system of medicine since ancient times. The extracts of ashwagandha are not mentioned in Ayurveda, and the content of phytochemicals in such extracts is higher compared to raw powder. Animal toxicity studies with such extracts may help to establish safe doses in humans. A subacute toxicity study with hydroalcoholic extract of ashwagandha showed no evidence of toxic effect or mortality in Wistar rats [15] Similarly, hydro alcoholic extract of ashwagandha was found to be safe in a sub-acute toxicity study involving rats and mice [16]. However the content of glycowithanolides and alkaloids in these hydro alcoholic extracts are not reported.

Owing to the presence of some toxic alkaloids in the root powder and crude extracts, safety of the commercially available extracts has been raised [7]. In few cases, it has been reported that ingestion of ashwagandha might cause stomach upset, diarrhea, and vomiting [2]. The alkaloid rich part of commercial ashwagandha extract was found to be toxic in a zebrafish embryo acute toxicity test as per OECD guideline 236 whereas detoxified ashwagandha extract (rich in glycowithanolides and containing very less alkaloids) was found to be non-toxic under the conditions of the study (under publication).

In the assessment and evaluation of the toxic characteristics of a test substance, determination of acute oral toxicity study in small animals (mice/rats) is usually an initial step. Results of acute toxicity obtained in this study clearly indicate that the PAE is acutely safe up to $2000 \mathrm{mg} / \mathrm{kg}$ bw in rats. The LD50 for rats was found to be $>2000 \mathrm{mg} / \mathrm{kg}$ bw which suggests that it is extremely safe. The $90 \mathrm{~d}$ repeated dose toxicity study in rats further supports the lack of toxicity of test sample as the repeated administration of PAE for $90 \mathrm{~d}$ to rats at the dosage level of $1000 \mathrm{mg} / \mathrm{kg}$ bw did not induce any observable toxic effects, when compared to its corresponding control group of animals. The biochemical and hematological parameters were normal. The histopathological observations of all the major organs were also normal. Converting rat dose to human equivalent dose, the $1000 \mathrm{mg} / \mathrm{kg}$ bw in rats corresponds to about $11 \mathrm{~g}$ daily in human [17].

\section{CONCLUSION}

The present study clearly indicates that PAE has no adverse/toxic effects in animal models at tested dosages as per OECD guidelines. It can be concluded that PAE is non-toxic at tested dose levels as evidenced by acute and repeated dose toxicity studies.

\section{AUTHORS CONTRIBUTIONS}

$\mathrm{BA}, \mathrm{MB}$ and BTK were involved in study concept and design; AS was involved in preparation and analysis of extract; NKG and SJ were involved in acquisition of data, analysis and interpretation. All the authors were involved in drafting and revising the manuscript.

\section{CONFLICT OF INTERESTS}

The authors declare that there is no conflict of interest

\section{REFERENCES}

1. Chandrasekhar K, Kapoor J, Anishetty S. A prospective, randomized double-blind, placebo-controlled study of safety and efficacy of a high-concentration full-spectrum extract of ashwagandha root in reducing stress and anxiety in adults. Indian J Psychol Med 2012;34:255-62.

2. Singh N, Bhalla M, Jager PD, Gilca M. An overview on ashwagandha: a rasayana (rejuvenator) of ayurveda. Afr J Tradit Complement Altern Med 2011:8:208-13.

3. Jassal PS, Kaur G. Comparative analysis of antioxidant activity and phytochemical contents in ethanolic leaf extracts of in vitro and field grown Withania somnifera. Asian J Pharm Clin Res 2016;9:239-44.

4. Chaudhuri D, Ghate NK, Sakar R, Mandal N. Phytochemical analysis and evaluation of antioxidant and free radical scavenging activity of Withania somnifera root. Asian J Pharm Clin Res 2012;5:193-9.

5. Panchal P, Singh K. Antimicrobial activity of withania somnifera and calotropis procera on pathogenic strains. Int J Curr Pharm Res 2015;7:76-8.

6. Shah PC, Trivedi NA, Bhatt JD, Hemavathi KG. Effect of Withania somnifera on forced swimming test induced immobility in mice and its interaction with various drugs. Indian J Physiol Pharmacol 2006;50:409-15.

7. European Food Safety Authority. Compendium of botanicals that have been reported to contain toxic, addictive, psychotropic or other substances of concern on request of EFSA. EFSA J 2009;7:281.

8. Blainski A, Lopes GC, De Mello JC. Application and analysis of the folin ciocalteu method for the determination of the total phenolic content from Limonium brasiliense L. Molecules 2013;18:6852-65.

9. Kamtekar S, Keer V, Patil V. Estimation of phenolic content, flavonoid content, antioxidant and alpha amylase inhibitory activity of marketed polyherbal formulation. J Appl Pharm Sci 2014;4:61-5. 
10. Kirk H, Sawyer R. Frait pearson chemical analysis of food. Edinburgh: Longman Scientific and Technical; 1998.

11. Cheok CY, Salman HAK, Sulaiman R. Extraction and quantification of saponins: a review. Food Res Int 2014;59:16-40.

12. Sreevidya N, Mehrotra S. Spectrophotometric method for estimation of alkaloids precipitable with dragendorff's reagent in plant materials. J AOAC Int 2003;86:1124-7.

13. Vador N, Vador B, Hole R. Simple spectrophotometric methods for standardizing ayurvedic formulation. Indian J Pharm Sci 2012;74:161-3.
14. Lowry $\mathrm{OH}$, Rosebrough NJ, Farr AL, Randall RJ. Protein measurement with the folin phenol reagent. J Biol Chem 1951;193:265-75.

15. Prabu PC, Panchapakesan S, Raj CD. Acute and sub-acute oral toxicity assessment of the hydroalcoholic extract of Withania somnifera roots in wistar rats. Phytother Res 2013;27:1169-78.

16. Sharada A, Solomon F, Devi P. Toxicity of Withania somnifera root extract in rats and mice. Pharm Biol 1993;31:205-12.

17. Nair AB, Jacob S. A simple practice guide for dose conversion between animals and human. J Basic Clin Pharm $2016 ; 7: 27-31$ 